\title{
Successful Endovascular Treatment of Aortoiliac Bifurcation Stenosis Using an Empirically Based T and Protrude-Stenting with Self- and Balloon-Expandable Stents
}

\author{
KEN-ICHIRO SASAKI, HIDETOSHI CHIBANA, TAKAFUMI UENO, NAOKI ITAYA, \\ MASAHIRO SASAKI AND YOSHIHIRO FUKUMOTO \\ Department of Cardio-Vascular Medicine, Kurume University School of Medicine, Kurume 830-0011, Japan \\ Received 5 April 2016, accepted 19 July 2016 \\ J-STAGE advance publication 6 February 2017
}

Edited by HIROYUKI TANAKA

\begin{abstract}
Summary: A 73-year-old woman with arteriosclerosis obliterans (ASO) was underwent a crossover stenting for an aortoiliac bifurcation from the right common iliac artery (CIA) with a self-expandable bare-metal stent (SE-BMS); however, a new stenosis later occurred just behind the bifurcation of the left CIA. An ex vivo experiment demonstrated that culotte-stenting by additional implantation of a balloon-expandable bare-metal stent (BE-BMS) through stent struts of the SE-BMS would be empirically infeasible. Although we had initially planned a T-stenting for the additional implantation of a BE-BMS in the left CIA, we finally deployed the stent in the CIA with the proximal end protruding into the previously-implanted SE-BMS through the stent struts to avoid incomplete coverage of the stenosis by reference to the ex vivo experiments. The patient has had no recurrence for 36 months.
\end{abstract}

Key words arteriosclerosis obliterans, culotte-stenting, T-stenting, ex vivo test

\section{INTRODUCTION}

Endovascular treatment (EVT) by stent implantation has become a conventional therapy for type-A stenosis in the TASC classification of aortoiliac lesions [1]. Although simultaneous-kissing stent reconstruction of aortoiliac bifurcation with a self-expandable baremetal stent (SE-BMS) and a balloon-expandable baremetal stent (BE-BMS) has been reported as an effective strategy to treat atherosclerotic stenoses of the bifurcation [2], there are few reports of additional Tstenting of a BE-BMS onto the struts of a previouslyimplanted SE-BMS for reconstruction of a bifurcation with atherosclerotic stenoses. Moreover, there is no report of a culotte-stenting of BE-BMS through the struts of the previously implanted SE-BMS to treat the bifurcation stenosis. Here we demonstrate the infeasi- bility of the culotte stenting ex vivo and then report a successful T and Protrude-, but not T-, stenting to treat the stenosis by reference to the ex vivo experiments.

\section{CASE REPORT}

A 73-year-old woman, who had undergone medical treatment for hypertension and diabetic mellitus for 11 years, complained of intermittent claudication of the right lower-limb. The ankle-brachial pressure index (ABI) of the right and left lower-limbs were 0.6 and 0.97 , respectively. The angiogram showed severe atherosclerotic stenosis of the right common iliac artery (CIA) (Fig. 1-A) and total occlusion of the right internal iliac artery. We diagnosed arteriosclerosis obliterans (ASO) and performed an EVT for the stenosis, which was classified as TASC A-type lesion [1]. After

Corresponding Author: Ken-ichiro Sasaki, MD, Ph. D., Division of Cardio-Vascular Medicine, Department of Internal Medicine, Kurume University School of Medicine, 67 Asahi-machi, Kurume, 830-0011, Japan. Tel: +81-942-31-7562, Fax: +81-942-33-6509, E-mail: sasaken@med.kurume-u.ac.jp

Abbreviations: ABI, ankle-brachial pressure index; BE-BMS, balloon-expandable bare-metal stent; CIA, common iliac artery; EVT, Endovascular treatment; IVUS, intravascular ultrasound; SE-BMS, self-expandable bare-metal stent. 

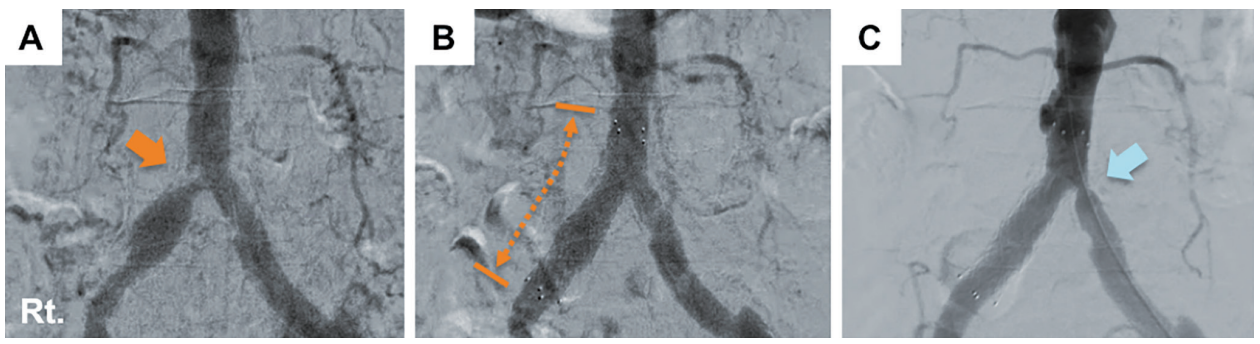

Fig. 1. Angiographic photos of abdominal and common iliac arteries in the first EVT

(A) The orange arrow indicates a severe atherosclerotic stenosis in the bifurcation of the right CIA, (B) The orange dotted arrow means the range of a SE-BMS implanted for the stenosis, (C) The light blue arrow indicates a severe stenosis in the bifurcation of the left CIA 7 months after the previous stenting.
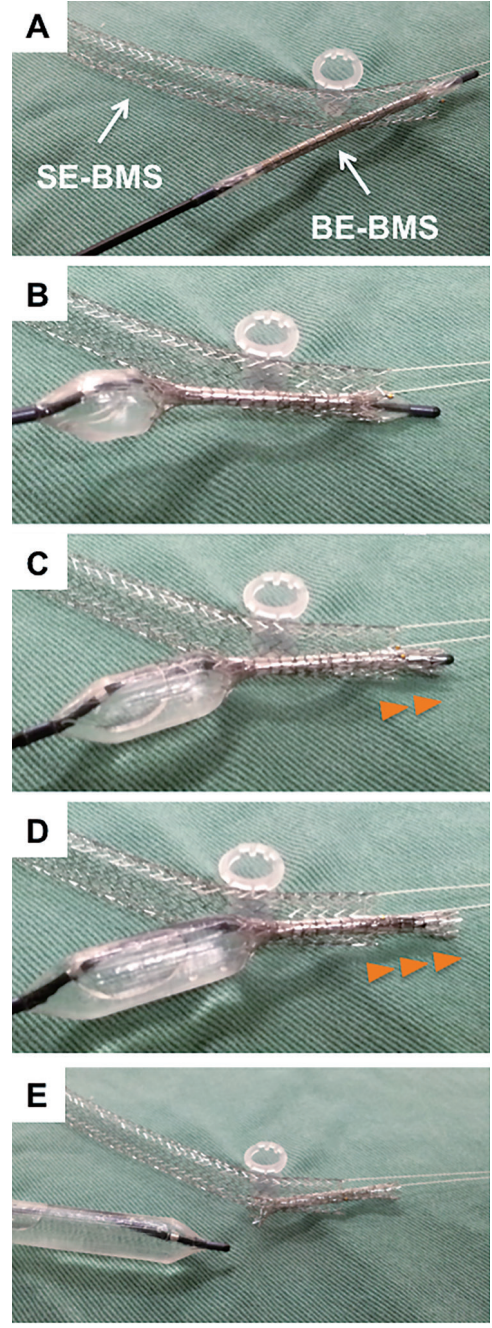

Fig. 2. Photos of an ex vivo experiment to test the possibility of the culotte-stenting with SE- and BE-BMSs (A) An expanded SE-BMS was fixed at the bilateral edge by manufactured silks and the SE-BMS was in part made the figure curved along the sidewall of a plastic tube. This situation was empirically mimicked the aortoiliac bifurcation where was implanted SE-BMS. A non-deployed BE-BMS was put through the strut of an expanded SE-BMS for trying an empirically culotte stenting. (B) A balloon on the BE-BMS began to deploy from the near side; however, the center and the far side of the balloon were not inflated. (C) The incompletely inflated balloon began to push the non-expanded BE-BMS forward and to do out of the way through the expanded SE-BMS (two orange head arrows). (D) The non-expanded BE-BMS was further pushed out of the away from the expanded-SE-BMS (three orange head arrows). (E) The balloon mounted on the BE-BMS was finally inflated all but the inflated-balloon was off the stent and moved to the near side, resulting that the non-expanded BE-BMS remained in the expanded SE-BMS. dilation of the stenosis with an $8.0 \times 40 \mathrm{~mm}$ balloon catheter (Jackal RX, Kaneka Medical, Osaka, Japan), a part of the atherosclerotic plaque shifted to the abdominal aorta through the bifurcation of the right CIA, but not into the left CIA. Accordingly, we performed a crossover stenting across the bifurcation of the left
CIA with a $10 \times 40 \mathrm{~mm}$ SE-BMS (Zilver 518 , Cook Medical, Bloomington, USA) to fully cover the shifted plaque (Fig. 1-B). The stenting did not impair the blood inflow to the left lower limb and again there was no atherosclerotic plaque shift into the left CIA. The $\mathrm{ABI}$ of the right and left lower-limbs recovered to 1.10 

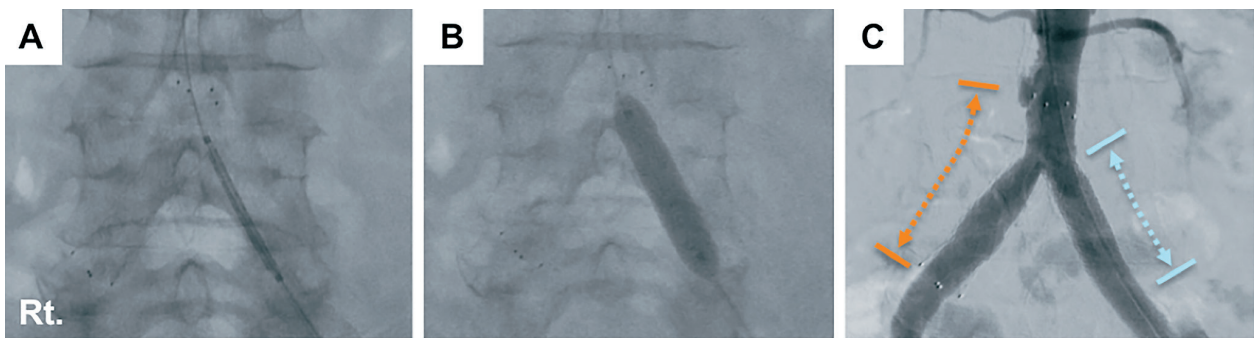

Fig. 3. Angiographic photos of abdominal and common iliac arteries in the second EVT (A) A non-deployed BE-BMS was delivered next to the wall of the previously-implanted SE-BMS from distal of the left CIA, (B) The BE-BMS was deployed in the left CIA with the proximal end protruding in the previouslyimplanted SE-BMS through the stent struts, (C) The orange dot arrow and lines indicate the range of a previously-implanted SE-BMS. The light blue dot arrow and lines do the range of an additionally-implanted BE-BMS.

and 1.02 , respectively. The intermittent claudication of the right lower-limb disappeared. However, at 7 months after the stenting, intermittent claudication within a walking distance of 200 meters newly appeared in the left lower-limb, but not in the right. The ABI of the right and left lower-limbs were 0.98 and 0.86 , respectively. The angiogram showed no stenosis in the previously implanted SE-BMS of the right lower-limb but revealed severe stenosis in the bifurcation of the left CIA (Fig. 1-C). The stenosis existed just behind the bifurcation next to the stent wall.

To evaluate suitable strategies to treat the stenosis, we empirically tested how deformations would occur in the stent structure after a dilation of the stent struts with an $8.0 \times 20 \mathrm{~mm}$ balloon catheter (Jackal RX, Kaneka Medical, Osaka, Japan). The cross-sectional balloon size of $8.0 \mathrm{~mm}$ corresponded to the cross-sectional size of the left CIA. The stent struts were initially dilated within the cross-sectional balloon size and resulted in the deformation of the stent structure. However, the deformation immediately restored to its former state after deflation of the balloon due to the shape-memory effect of the stent. Next, we empirically checked whether a culotte-stenting by an additional implantation of an $8.0 \times 27 \mathrm{~mm}$ BE-BMS (Express LD, Boston Scientific, Natick, USA) through stent struts of the previously implanted SE-BMS (Fig. 2-A) would be feasible. The balloon of the BE-BMS gradually began to inflate in the struts of a deployed SE-BMS, which was the same as the previously implanted SE-BMS, from the proximal side of the catheter. However, the stent did not expand at all (Fig. 2-B). The non-expanded stent shifted off balloon and moved forward in the SE-BMS (Fig. 2-C and 2-D). The entirely inflated stent balloon was pushed out of the struts of the SE-BMS (Fig. 2-E). These results sug- gested that a T-stenting would be more suitable than a culotte-stenting to treat the stenosis by the additional implantation of a BE-BMS into the previously implanted SE-BMS.

After the above preliminaries, we performed the planned EVT via the left femoral artery. A 0.018-inch guidewire (Treasure, Asahi Intec, Aichi, Japan) was advanced into the aortic aorta through the stenosis of the left CIA and the struts of the previously implanted SE-BMS. We dilated the stenosis with a balloon (Jackal RX, Kaneka Medical, Osaka, Japan) and implanted the BE-BMS next to the wall of the previously implanted SE-BMS with a T-stenting technique. At the stent implantation, we deployed the BE-BMS in the left CIA with the proximal end protruding into the previously-implanted SE-BMS through the stent struts within a few millimeters above the aortoiliac bifurcation to avoid incomplete coverage of the stenosis (Fig. 3-A, 3-B, and 3-C). Intravascular ultrasound (IVUS) (Visions PV.018, Volcano, San Diego, USA) showed that a part of proximal edge of the implanted BE-BMS was located in the struts of the previously implanted SE-BMS and that the BE-BMS fully covered the stenosis without any stent deformation. The ABI of the right lower-limb recovered to 1.05 and the intermittent claudication of the limb disappeared, indicating successful EVT for the limb. At 9 months after the EVT, the angiographic success was maintained on angiography and there was no significant pressure gradient $(<10 \mathrm{mmHg}$ ) between the aorta and the left CIA. The ABI of both left and right lower-limbs have been maintained at more than 1.0 with no recurrence of intermittent claudication as of 36 months after the final treatment. 


\section{DISCUSSION}

In this case, the patient had intermittent claudication of the right lower-limb due to atherosclerotic obliterans and the symptoms markedly restricted her daily life. Although supervised exercise training is recommended as an initial treatment modality for peripheral arterial disease patients with intermittent claudication in the ACC/AHA Guidelines [1], consistent lower back pain prevented the patient from doing exercise training. Therefore, we performed EVT for the right CIA type-A stenosis in this patient. Although SE-BMS implantation to the CIA was successful with no problem in the angiographic finding for the aortoiliac bifurcation after the treatment, newly progressive stenosis unexpectedly occurred in the bifurcation, which induced intermittent claudication of the contralateral lower-limb. Although we did not perform additional balloon angioplasty for the aortoiliac bifurcation using a kissing-balloon technique, atherosclerotic plaque might have shifted into the contralateral CIA ostium at the SE-BMS implantation. However, we have no diagnostic imaging of IVUS after the implantation.

For treatment of the newly progressive stenosis, we considered that an additional culotte-stenting of SE-BMS or BE-BMS to the previously implanted SEBMS might be desirable to avoid incomplete coverage of the aortoiliac bifurcation stenosis. BE-BMS and SE-BMS are usually made of stainless steel and Nitinol, respectively. Nitinol, which is an alloy of nickel and titanium, has an ability through thermal memory to keep its original shape above a preset temperature. BE-BMSs exert greater radial force than SE-BMSs, while SE-BMSs have greater elasticity than BE-BMSs. The radial force of BE-BMS prevents the recoil of the deployed stent, and the elasticity of SE-BMS allows the deployed stent easily to regain its original shape after a pressure deformation, helping to prevent lumen loss after stent implantations. In addition, the outward force acting on the vessel wall is much lower in nitinol stents than stainless steel ones, suggesting that SEBMSs reduce continuous intimal injury at the stent implanting vessel wall and prevent neointimal hyperplasia that can cause in-stent restenosis. Nevertheless, a newly progressive stenosis developed next to the SE-BMS wall in the aortoiliac bifurcation and expanded into the left CIA in this case. It was reported that BE-BMSs were appropriate for implantation to rigid and straight lesions [3] and that they have higher radiopacity facilitating precise stent placement [4]. Accordingly, we considered that an additional culottestenting of BE-BMS to the previously implanted SE-
BMS might be a desirable strategy for treating the newly progressive stenosis.

Because it was unclear whether the strategy would be feasible, we empirically tested the strategy beforehand. Contrary to our expectation, the retention of stent struts of the SE-BMS was stronger than the radial force of a fully-dilated non-compliant balloon and a deployed BE-BMS, indicating that an additional culotte-stenting of BE-BMS to the previously implanted SE-BMS must lead to unrepairable deformation of the BE-BMS and occlusion of the left CIA, and that the additional T-stenting of BE-BMS for treating the newly progressive stenosis would be desirable for the treatment. We initially planned simple T-stenting with no contact between the SE-BMS and BE-BMS. However, in order to avoid incomplete coverage of the aortoiliac bifurcation stenosis, we finally deployed a BE-BMS with the proximal end protruding a few millimeters into the previously implanted SE-BMS through the stent struts, by reference to observations made during the ex vivo experiments which indicated that the BE-BMS deployment would be feasible. This technique was previously reported as a $\mathrm{T}$ and protrudestenting technique, or TAP-stenting in short. Midulla et al. reported in 23 patients with aortoiliac bifurcation stenoses that percutaneous transluminal angioplasty by an implantation of SE-BMS and BE-BMS simultaneously using the TAP-stenting technique brought desirable clinical outcomes for patients with no complications but high patency rate at a mean 16.3 months after the treatment [5]. Our present patient had a similar desirable clinical course with no recurrence 36 months after an additional TAP-stenting with BEBMS. Nevertheless, when treating aortoiliac bifurcation stenoses with other SE-BMS and BE-BMS, it will be necessary to test ex vivo to determine whether the treatment strategy is feasible.

\section{CONCLUSION}

The results of our empirical feasibility tests demonstrated that a culotte-stenting of SE-BMS or BE-BMS for a previously implanted SE-BMS was not practicable. As an alternative strategy for treating an aortoiliac bifurcation lesion, the TAP-stenting technique may be feasible for an additional implantation of a BE-BMS to a newly progressive stenosis next to a previously implanted SE-BMS in the aortoiliac bifurcation. However, a large-scale clinical study is needed to assess whether this treatment strategy achieves desirable clinical courses and patency of the implanted stents in the long term. 


\section{Compliance with ethical standards}

The authors complied with human study guidelines of Kurume University Hospital and the patient provided informed consent for publication of this case report.

\section{Declaration of conflicting interest}

The authors declared no potential conflicts of interest with respect to the report, authorship, and/or publication of this article.

\section{Funding}

The authors received no financial support for the report, authorship, and/or publication of this article.

ACKNOWLEDGMENTS: The ex vivo experiments were performed by using complimentary balloon and stent catheters after the validity date had already expired. We thank Kaneka Medical, Cook Medical, and Boston Scientific that kindly provided the catheters for the experiments.

\section{REFERENCES}

1. Norgren L, Hiatt WR, Dormandy JA, Nehler MR, Harris KA et al. TASC II Working Group: Inter-Society Consensus for the Management of Peripheral Arterial Disease (TASC II). J Vasc Surg. 2007; 45:S5-S67.

2. Björses K, Ivancev K, Riva L, Manjer J, Uher P et al. Kissing stents in the aortic bifurcation--a valid reconstruction for aorto-iliac occlusive disease. Eur J Vasc Endovasc Surg. 2008; 36:424-431.

3. Duda SH, Wiskirchen J, Tepe G, Bitzer M, Kaulich TW et al. Physical properties of endovascular stents: an experimental comparison. J Vasc Interv Radiol. 2000; 11:645-654.

4. Dyet JF, Watts WG, Ettles DF, and Nicholson AA. Mechanical properties of metallic stents: how do these properties influence the choice of stent for specific lesions? Cardiovasc Intervent Radiol. 2000; 23:47-54.

5. Midulla M, Martinelli T, Goyault G, Lions C, Abboud G et al. T-stenting with small protrusion technique (TAPstenting) for stenosed aortoiliac bifurcations with small abdominal aortas: an alternative to the classic kissing stents technique. J Endovasc Ther. 2010; 17:642-651. 